\title{
3-Dimensional CT Lymphography in Identifying the Sentinel Node in Breast Cancer
}

\author{
Junko Honda ${ }^{1}$, Chieko Hirose ${ }^{2}$, Masako Takahashi ${ }^{3}$, Sonoka Hisaoka², \\ Miyuki Kanematsu ${ }^{1}$, Yoshimi Bando ${ }^{4}$ and Mitsunori Sasa ${ }^{5}$ \\ ${ }^{1}$ Department of Surgery, National Hospital Organization Higashitokushima \\ Medical Center, 1-1, Ohmukai-kita, Ootera, Itano, Tokushima, \\ ${ }^{2}$ Department of Radiology, National Hospital Organization Higashitokushima \\ Medical Center, 1-1, Ohmukai-kita, Ootera, Itano, Tokushima, \\ ${ }^{3}$ Department of Radiology, Tokushima Breast Care Clinic, \\ Nakashimada-Cho, Tokushima, \\ ${ }^{4}$ Department of Molecular and Environmental Pathology, \\ Institute of Health Biosciences, The University of Tokushima Graduate \\ School, Kuramoto-Cho, Tokushima, \\ ${ }^{5}$ Department of Surgery, Tokushima Breast Care Clinic, \\ Nakashimada-Cho, Tokushima, \\ Japan
}

\section{Introduction}

Sentinel node biopsy (SNB) has become a standard surgical procedure for patients with earlystage breast cancer. The sentinel nodes (SN) can be identified by a double-mapping procedure based on a gamma probe-guided method and a dye-guided method using a radioisotope (RI), or by a triple-mapping procedure that includes lymphoscintigraphy and is even more effective. However, the RI method can be performed only at institutions that are trained and licensed to use RI, and other institutions must rely on dye methods alone for SN identification. On the other hand, to obtain images of the lymph vessels and nodes, indirect lymphography seems to be a more convenient than direct intralymphatic administration of a contrast medium. Several studies of indirect lymphography were reported in the 1980s, and Suga et al. reported successfully identifying SN by 3-dimensional computed tomographic lymphography (CTLG) using a nonionic contrast medium. We have also been identifying $\mathrm{SN}$ in breast cancer patients by CTLG and a dye-guided method since February 2003 as a clinical trial. Here, we report our findings to date regarding the clinical efficacy and problems associated with $\mathrm{SN}$ identification by the combination of CTLG and a dye-guided method.

\section{Patients and methods}

All studies in this paper were approved by the ethics committee of National Hospital Organization Higashitokushima Medical Center. After presenting a detailed explanation of this clinical trial, written informed consent was obtained from all patients. 


\subsection{Patients}

During the period from February 2003 through March 2007, 218 Japanese patients with T1N0M0 or T2N0M0 primary breast cancer were treated at the Tokushima Breast Care Clinic. SN identification was performed by combined application of CTLG and a dye-guided method. In two of the patients the CTLG was performed before and after excisional biopsy, and in one patient with bilateral disease CTLG was performed on both sides. Thus, CTLG was performed 221 times in total, while the dye-guided method was performed a total of 219 times. In principle, backup dissection was performed for patients found to be SN metastasis-positive provided that informed consent was granted. For metastasis-negative patients, axillary dissection was omitted on the basis of informed consent.

\subsection{Methods}

CTLG was performed as previously described. Each patient was placed in the supine position, with the arms positioned upward but bent at the elbow with the hands at the side of the cranium. This position is similar to the surgical position. First, plain CT scanning of the affected axilla was carried out using a high-speed FX/i single-detector helical CT scanner (GE Yokogawa Medical Systems, Tokyo, Japan). The X-ray beam thickness was $3 \mathrm{~mm}$ and the pitch was 1.5. Then, one $\mathrm{mL}$ of iopamidol (Iopamiron 300; $300 \mathrm{mgI} / \mathrm{ml}$, Schering, Osaka, Japan) was injected subcutaneously (or intradermally) to the areola or both subcutaneously (or intradermally) to the areola and subcutaneously (or intradermally) above the tumor for a few minutes with a 26-gauge 5/8-inch hypodermic needle attached to a tuberculin syringe (Figure 1a). One minute after the injection, axillary CT scanning (CT lymphography) was done with the same thickness and pitch as described above. In the case that the enhancement of the lymph vessels and lymph nodes was poor, the site(s) where the contrast medium had been injected was gently massaged, and after 3-5 min the CT was repeated. Finally, the remaining medium (about $99 \mathrm{ml}$ ) was intravenously administered from the unaffected forearm to examine distal metastases. A CT scanning was done in the thoracic and upper abdominal areas with $7 \mathrm{~mm}$ beam thickness and 1.5 pitch in the axillary region with $3 \mathrm{~mm}$ beam thickness and 1.5 pitch. 2dimensional images of axilla (Figure 1b) were reconstructed at intervals of $1 \mathrm{~mm}$, and then 3-dimensional images were created by the volume-rendered method to correlate with the surgical view (Figure 1c). SNs were predicted from CT images by identification of enhanced lymph vessels and/or lymph nodes and assessment of the CT values. The CT values of lymph nodes were measured at their maximally enhanced point. If an $\mathrm{SN}(\mathrm{s})$ was identified, a mark was made on the skin immediately above the SN by using an oil painting pen (Figure 1d).

The dye-guided method was also performed as previously described. Briefly, 2 3 $\mathrm{mL}$ of indigo carmine blue was injected subcutaneously (or intradermally) to the areola, followed by massage of the injection site for a few minutes. Just before performing an operation, we used ultrasonography to confirm the location of $\mathrm{SN}(\mathrm{s})$ that has been identified by CTLG. Five to 15 min later a skin incision was made at the site(s) that were marked in the CTLG, and any blue-stained lymph vessels and lymph nodes were identified. If no SN(s) was identified by either the dye-guided method or CTLG, axillary lymph node dissection was performed. 

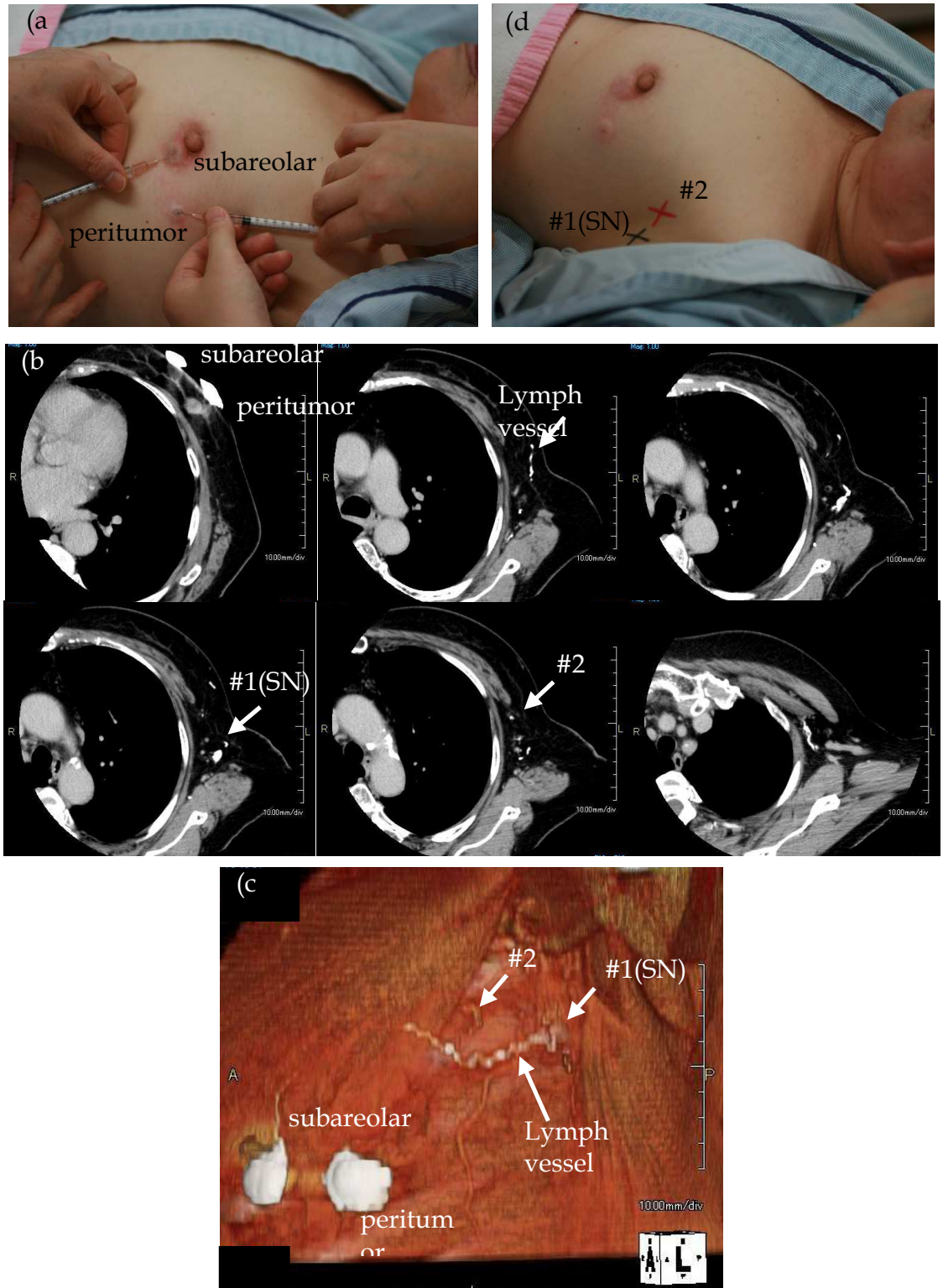

Fig. 1. Method for identification of the SNs performed by CTLG. Images in patient with left breast cancer. (a) One $\mathrm{ml}$ of iopamidol was injected both subcutaneously (or intradermally) to the areola and subcutaneously (or intradermally) above the tumor. (b) Axial image demonstrating a tortuous, enhanced lymph vessel that reaches SN. (c) 3-dimensional CT lymphography image clearly showing an enhanced lymh vessel and an opacified SN (\#1). (d) The mark was made on the skin immediately above the identificated SN by using an oil painting pen. 


\subsection{Definition of SNs}

CTLG: A lymph node was defined as an SN if visual inspection or the CT value (the increase of Hounsfield units after subcutaneous injection) confirmed it to be enhanced or if it was confirmed to connect to an enhanced lymph vessel.

Dye-guided method: A lymph node was defined as an SN if it was dyed blue or if it was confirmed to connect to a blue lymph vessel.

\subsection{SN identification rates and the clinicopathological findings}

The results were analyzed in relation to the success/failure of SN identification and various clinicopathological parameters, including the patient age, menopausal status, body mass index (BMI), tumor diameter, presence/absence of excisional biopsy, histological type, presence/absence of lymph node metastasis and presence/absence of vascular invasion.

\subsection{Statistical analysis}

The relationships between the success/failure of $\mathrm{SN}$ identification and the clinicopathological findings were analyzed for statistical significance using the chi-square test. A p value of $<0.05$ was considered to indicate statistical significance.

\section{Results}

\subsection{SN identification rates by CTLG and the dye-guided method}

Identification of the $\mathrm{SN}(\mathrm{s})$ was achieved in $212(96 \%)$ of the total 221 performances of CTLG. The number of detected SNs ranged from 1 to 3, with a mean of 1.2 SNs. With the dyeguided method the identification rate was $92 \%$ (202/219 tests). The combined identification rate was $99 \%$ (Table 1$)$.

\begin{tabular}{cccc}
\hline & Dye & CTLG & Combination \\
Lymph vessels & $212(97 \%)$ & $202(91 \%)$ & $215(98 \%)$ \\
Sentinel nodes & $202(92 \%)$ & $212(96 \%)$ & $216(99 \%)$ \\
\hline
\end{tabular}

Dye : Dye-guided method, CTLG : CT Lymphography, Combination : Combination of CTLG and Dye

Table 1. Identification rates of lymph vessels and sentinel nodes

With CTLG, both the lymph vessels and lymph nodes were clearly enhanced and the SNs could be identified in 189 patients $(86 \%)$. In 5 patients $(2 \%)$ the lymph vessels were not enhanced but the lymph nodes were, and the SNs could be identified. In 7 other patients $(3 \%)$, the lymph vessels were enhanced while the lymph nodes were not clearly enhanced, but the SNs could be identified from the CT value. In 5 patients (2\%) neither the lymph vessels nor the lymph nodes were enhanced, but the SNs could be identified from the CT value. In 6 patients (3\%) the lymph vessels were enhanced but the lymph nodes were not, but the SNs could be identified on the basis of confirmation of their connection to the enhanced lymph vessels. Finally, in 9 patients (4\%) neither the lymph vessels nor the lymph nodes were enhanced, and SNs could not be demonstrated (Table 2). 


\begin{tabular}{ccc}
\hline Lymph vessels & Node & \\
Enhanced & Cleary enhanced & $189(86 \%)$ \\
Unenhanced & Cleary enhanced & $5(2 \%)$ \\
Enhanced & Assessment of CT values & $7(3 \%)$ \\
Unenhanced & Assessment of CT values & $5(2 \%)$ \\
Enhanced & Unenhanced & $6(3 \%)$ \\
Unenhanced & Unenhanced & $9(4 \%)$ \\
\hline
\end{tabular}

Table 2. Findings of CT lymphography $(n=221)$

\subsection{SN identification rates and the clinicopathological findings}

Analysis of the relationships between the SN identification rates and the clinicopathological findings showed that the SN identification rates with CTLG, the dye-guided method and the combined method showed no differences as a function of the age, menopausal status, tumor diameter or histopathological type. However, with the dye-guided method the SN identification rate was significantly lower in patients with a BMI of 25 or higher, whereas with CTLG and the combined method the SN identification rate was not influenced by the BMI. With CTLG and the combined method, the SN identification rate was significantly lower in the node-positive patients, and with the combined method it was significantly lower in patients with vascular invasion. In addition, in the patients who had undergone lateral-upper region excisional biopsy the SN identification rate with CTLG was lower than in the patients who had undergone excisional biopsy in a region other than the lateral-upper region, although the difference did not reach statistical significance. Moreover, with the dyeguided method the SN identification rate was lower in the patients who had undergone excisional biopsy, regardless of the region, compared with the patients who had not undergone excisional biopsy (Table 3).

\begin{tabular}{cccc}
\hline & Dye $(+), \mathrm{n}=202$ & $\begin{array}{c}\text { CTLG }(+), \\
\mathrm{n}=212\end{array}$ & Combination $(+), \mathrm{n}=216$ \\
Age (years) & & & \\
$\leq 35$ & $6(100 \%)$ & $6(100 \%)$ & $6(100 \%)$ \\
$36-50$ & $87(90 \%)$ & $92(95 \%)$ & $96(98 \%)$ \\
$\geq 51$ & $109(94 \%)$ & $114(97 \%)$ & $114(98 \%)$ \\
Menopausal state & $103(92 \%)$ & $108(96 \%)$ & $105(98 \%)$ \\
Pre & $99(93 \%)$ & $104(95 \%)$ & $111(99 \%)$ \\
Post & & & \\
BMI & $160(95 \%) \#$ & $164(96 \%)$ & $166(98 \%)$ \\
$<25$ & $42(84 \%)$ & $48(96 \%)$ & $50(100 \%)$ \\
$\geq 25$ & & & $197(99 \%)$ \\
Tumor size (cm) & $183(92 \%)$ & $193(96 \%)$ & $19(100 \%)$ \\
$\leq 2.0$ & $19(100 \%)$ & $19(100 \%)$ & \\
$>2.0$ & & &
\end{tabular}




\begin{tabular}{|c|c|c|c|}
\hline & Dye $(+), n=202$ & $\begin{array}{c}\text { CTLG (+), } \\
n=212\end{array}$ & Combination $(+), \mathrm{n}=216$ \\
\hline \multicolumn{4}{|l|}{ With excisionary biopsy * } \\
\hline In lateral-upper region & $17(89 \%)$ & $18(95 \%)$ & $19(100 \%)$ \\
\hline In other regions & $46(88 \%)$ & $52(100 \%)$ & $52(100 \%)$ \\
\hline \multicolumn{4}{|l|}{ Histological type } \\
\hline IIa1 & $71(88 \%)$ & $80(99 \%)$ & $80(99 \%)$ \\
\hline IIa2 & $22(100 \%)$ & $23(100 \%)$ & $22(100 \%)$ \\
\hline IIa3 & $64(96 \%)$ & $63(94 \%)$ & $65(97 \%)$ \\
\hline IIb3 & $8(89 \%)$ & $8(89 \%)$ & $9(100 \%)$ \\
\hline DCIS & $13(100 \%)$ & $12(92 \%)$ & $13(100 \%)$ \\
\hline Others & $24(89 \%)$ & $26(93 \%)$ & $27(100 \%)$ \\
\hline \multicolumn{4}{|l|}{ Nodal status } \\
\hline n $(-)$ & $177(93 \%)$ & 189 (98\%) \# & $191(100 \%) \#$ \\
\hline $\mathrm{n}(+)$ & $25(89 \%)$ & $23(82 \%)$ & $25(89 \%)$ \\
\hline \multicolumn{4}{|l|}{ Vascular invasion } \\
\hline $\mathrm{v}(-)$ & $160(91 \%)$ & $172(97 \%)$ & $174(99 \%) \#$ \\
\hline $\mathrm{v}(+)$ & $42(95 \%)$ & $40(91 \%)$ & $42(95 \%)$ \\
\hline
\end{tabular}

BMI : Body mass index, Dye : Dye-guided method, CTLG : CT Lymphography, Combination : Combination of CTLG and Dye, \# ; $<<0.05,{ }^{*}$; cases with excisional biopsy ( $\mathrm{n}=71$ )

Table 3. Sentinel node(s) identification rate and clinicopathological findings (all cases) (Dye $\mathrm{n}=219$, CTLG $\mathrm{n}=221$, Combination $\mathrm{n}=219$ )

\subsection{Size of metastasis and the SN identification rate in node-positive patients}

The patients found to be positive for metastasis were classified and analyzed on the basis of the size of metastasis: $<0.2 \mathrm{~mm}, 0.2 \mathrm{~mm} \sim 2 \mathrm{~mm}$ and $>2 \mathrm{~mm}$. The results showed that the SN identification rate by both CTLG and the dye-guided method decreased as the size of metastasis increased, although the difference did not reach statistical significance (Table 4).

\begin{tabular}{ccc}
\hline Metastatic lesion size & Dye $(+)$ & CTLG $(+)$ \\
Isolated tumor cells $(<0.2 \mathrm{~mm})$ & $2(100 \%)$ & $2(100 \%)$ \\
Micrometastasis $(0.2-2 \mathrm{~mm})$ & $10(91 \%)$ & $9(82 \%)$ \\
Macrometastasis $(\geq 2.0 \mathrm{~mm})$ & $13(87 \%)$ & $12(80 \%)$ \\
\hline
\end{tabular}

Dye : Dye-guided method, CTLG : CT Lymphography

Table 4 . Size of the metastatic lesion in the node positive cases $(n=28)$

\subsection{Number of lymph vessels leading to SNs and the number of SNs}

In this study, we were able to analyze the data on the lymph vessels leading to SNs and the number of SNs of 200 patients. A single route with a single SN was the most common pattern, seen in $68 \%$ of the patients. Multiple routes with a single SN were detected in $4 \%$ of the patients, while a single route with multiple SNs was seen in $10 \%$ and multiple routes with multiple SNs were seen in $8 \%$. Overall, multiple SNs were identified in $18 \%$ of the patients (Table 5). 


$\begin{array}{lc}\text { Single rout and single node } & 151(68 \%) \\ \text { Multi routs and single node } & 8(4 \%) \\ \text { Single rout and multi nodes } & 23(10 \%) \\ \text { Multi routs and multi nodes } & 18(8 \%)\end{array}$

Table 5. Number of lymph vessels leading to sentinel nodes and the number of sentinel nodes

\section{Discussion}

In breast cancer surgery, dissection of the axillary lymph nodes is considered effective for the objectives of performing staging and achieving local control. Therefore, in recent years SNB has become a standard procedure for patients with no metastasis of the axillary lymph nodes since it avoids unnecessary dissection. Combined use of a RI (gamma probe-guided method and lymphoscintigraphy) and a dye is currently considered to be more efficient for identification of the $\mathrm{SN}(\mathrm{s})$ than the single use of either of these methods. In Japan, there are many institutions that do not have the necessary facilities for using RI and thus must use the dye-guided method alone to identify SNs. On the other hand, Suga et al. proposed using a method called CTLG, which employs a nonionic contrast medium, and we have applied that method for SN identification since February 2003. In this paper we have reported our findings regarding the usefulness and problems associated with combined application of CTLG and the dye-guided method for SN identification.

Our experimental subjects were 219 Japanese patients with primary breast cancer that was thought to be clinically free of axillary lymph node metastasis. CTLG and the dye-guided method were employed in an attempt to identify the SN(s) in each of the patients. The SN identification rates were $96 \%$ with CTLG alone, 92\% with the dye-guided method alone and $99 \%$ when the findings with the two methods were combined. Those results are good when compared with the published data for combined use of an RI and the dye-guided method. In particular, our SN identification rate with the dye-guided method is better than the rates that have been reported to date. We think that this is because the location of the SN had already been determined by the CTLG, making it easy to identify the lymph vessels and lymph nodes. The breakdown of the identification by CTLG showed clear enhancing of the lymph nodes in $88 \%$ of the patients, while in $5 \%$ of the patients identification of the SN was possible on the basis of the CT value in spite of the fact that the lymph nodes were not clearly enhanced. Because the observations were macroscopic, when the dye-guided method was used alone, SN identification was difficult unless the lymph node was stained to a sufficient degree. With CTLG, on the other hand, even if the enhancement is not very striking it can be surmised that the SN identification rate will be improved since the CT value can be taken into consideration.

In 14 patients, the SN was identified by CTLG, but not by dye-guided method. The location of SN was confirmed by ultrasonography just before the operation in all of these patients. In these 14 patients, not only SN but also neighboring nodes were sampled at the same time. Therefore, it seemed that the SN biopsies had been performed accurately. 
The sensitivity of CTLG could not be investigated in our patient population because backup axillary dissection was not performed for patients who were metastasis-negative in the SNB. In addition, none of the patients experienced postoperative recurrence in the axillary lymph nodes, but that does not serve as a basis for claiming that there were few false-negatives. However, Tangoku et al. reported the sensitivity of CTLG to be $98 \%$, and for that reason it can be thought that the sensitivity of CTLG is not inferior to that of the RI method.

With regard to correlations between the clinicopathological findings and the $\mathrm{SN}$ identification rate, the patient age, the location of the tumor, whether or not surgical biopsy was performed, the tumor size and the presence/absence of lymph node metastasis have been reported to influence the identification of the SN. In our patient series, the results showed no differences as a function of the age, menopausal status, tumor diameter or histopathological type. However, with the dye-guided method the SN identification rate was significantly lower in patients with a BMI of 25 or higher, whereas with CTLG and combination method the $\mathrm{SN}$ identification rate was not influenced by the BMI. As the reason for this difference, it is noted that with the dye-guided method it can be difficult to discern staining of lymph vessels and nodes with the naked eye if considerable subcutaneous fat is present, whereas with CTLG the subcutaneous fat plays no role since the observation is done by $\mathrm{CT}$. In addition to the BMI, discrepancies between the $\mathrm{SN}$ identification rates with these three methods were observed as a function of the presence/absence of lymph node metastasis, the site of excisional biopsy (i.e., the lateral upper region and regions other than the lateral upper region), and the presence/absence of vascular invasion. Especially, the identification rates with CTLG and the combined method were significantly lower in nodepositive patients compared to node-negative patients, and significantly lower with the combined method in vascular invasion-positive patients compared to negative patients. The size of injected particles, the injected volume, the injection site have been reported as examination factors that can influence SN identification. The nonionic contrast media that are used in CTLG have a larger particle size than dyes. For that reason it can be hypothesized that, in patients with lymph node metastasis or vascular invasion and in patients who had undergone lateral upper region excisional biopsy, who can be predicted to have lymph vessel occlusion, the movement of a nonionic contrast medium would be impeded compared with that of a dye, thus resulting in a lower $\mathrm{SN}$ identification rate. On the other hand, the $\mathrm{SN}$ identification rate with the dye-guided method was lower in the patients with excisional biopsy in any region than in patients without excisional biopsy. It can be hypothesized that this is probably because excisional biopsy leads to edema of the connective tissue, which makes it difficult to distinguish the dye.

We also investigated the number of SNs and the number of lymph vessels leading to them. These evaluations are difficult to achieve by the gamma probe-guided method and the dyeguided method, but CTLG permits detailed investigation. Our results showed that there was a single route leading to a single $\mathrm{SN}$ in $68 \%$ of the patients, multiple routes leading to a single SN in $4 \%$, a single route leading to multiple SNs in $10 \%$ and multiple routes leading to multiple SNs in $8 \%$. These results are in agreement with those reported by Tangoku et al. Thus, approximately $18 \%$ of the patients in our series had multiple SNs, and it can be hypothesized that it would be difficult to biopsy all of them if only the dye-guided method were employed. We think that combined use of CTLG with the dye-guided method would 
permit accurate biopsy, and for that reason we anticipate that combined performance of CTLG will prove useful.

CTLG is a diagnostic test that is performed prior to surgery for breast cancer. Studies are warranted to determine whether the $\mathrm{CT}$ findings or performance of fine-needle aspiration cytology of the $\mathrm{SN}$ will make it possible to achieve diagnosis of $\mathrm{SN}$ metastasis preoperatively and then decide whether or not SNB should be performed. Such diagnosis leading to avoidance of unnecessary surgical procedures would represent a great clinical advantage by reducing the burden on the patient. Moreover, in the future it will be necessary to compare the usefulness of CTLG with the RI method.

\section{Conclusion}

Combined performance of CTLG with the dye-guided method permits better elucidation of the location of the $\mathrm{SN}(\mathrm{s})$ in breast cancer. This makes it easier to identify the $\mathrm{SN}(\mathrm{s})$ and results in a higher $\mathrm{SN}$ identification rate compared with application of the dye-guided method alone. Combination of CTLG and the dye-guided method was especially useful in obese patients. In addition, in patients with multiple SNs, it is advantageous to be able to perform SNB accurately. However, in patients with occlusion of the lymph vessels due to lymph node metastasis, having undergone lateral upper region lumpectomy or the presence of vascular invasion, there is a possibility that false-negative diagnostic results will be generated with CTLG.

\section{Acknowledgement}

Written consent was obtained from the patients or their relative for publication of this article.

\section{References}

Minato M, Hirose C, Sasa M, Nishitani H, Hirose Y \& Morimoto T. 3-dimensional computed tomography lymphography-guided identification of sentinel lymph nodes in breast cancer patients using subcutaneous injection of nonionic contrast medium: a clinical trial. Jornal of Computer Assisted Tomography, Vol.28, No.1, (JanuaryFebruary 2004), pp. 46-51

Suga K, Ogasawara N, Okada M \& Matsunaga N. Interstitial CT lymphography-guided localization of breast sentinel lymph node: preliminary results. Surgery, Vol.133, No.2, (February 2003), pp. 170-179

Suga K, Ogasawara N, Yuan Y, Okada M, Matsunaga N \& Tangoku A. Visualization of breast lymphatic pathways with an indirect computed tomography lymphography using a nonionic monometric contrast medium iopamidol: preliminary results. Investigative Radiology, Vol.38, No.2, (February 2003), pp. 73-84

Takahasi M, Sasa M, Hirose C, Hisaoka S, Taki M, Hirose T \& Bando Y. Clinical efficacy and problems with CT lymphography in identifying the sentinel node in breast cancer. World Journal of Surgical Oncology, Vol.6, No.57, (June 2008) 
Tangoku A, Yamamoto S, Suga K, Ueda K, Nagashima Y, Hida M, Sato T, Sakamoto K \& Oka M. Sentinel lymph node biopsy using computed tomographylymphography in patients with breast cancer. Surgery, Vol.135, No.3, (March 2004), pp. 258-265

Tangoku A, Seike J, Nakano K, Nagao T, Honda J, Yoshida T, Yamai H, Matsuoka H, Uyama K, Goto M, Miyoshi T \& Morimoto T. Current status of sentinel lymph node navigation surgery in breast and gastrointestinal tract. The Journal of Medical Investigation, Vol.54, No.1-2, (February 2007), pp. 1-18 


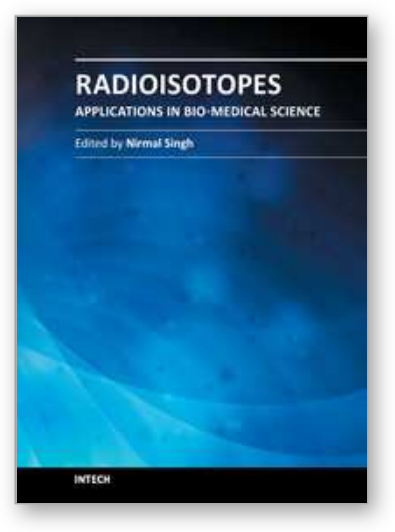

\author{
Radioisotopes - Applications in Bio-Medical Science \\ Edited by Prof. Nirmal Singh
}

ISBN 978-953-307-748-2

Hard cover, 320 pages

Publisher InTech

Published online 21, November, 2011

Published in print edition November, 2011

The book Radioisotopes - Applications in Bio-Medical Science contains two sections: Radioisotopes and Radiations in Bioscience and Radioisotopes and Radiology in Medical Science. Section I includes chapters on medical radioisotope production, radio-labeled nano-particles, radioisotopes and nano-medicine, use of radiations in insects, drug research, medical radioisotopes and use of radioisotopes in interdisciplinary fields etc. In Section II, chapters related to production of metal PET (positron emission tomography) radioisotopes, 3-dimensional and CT (computed tomography) scan, SS nuclear medicine in imaging, cancer diagnose and treatments have been included. The subject matter will by highly useful to the medical and paramedical staff in hospitals, as well as researchers and scholars in the field of nuclear medicine medical physics and nuclear biochemistry etc.

\title{
How to reference
}

In order to correctly reference this scholarly work, feel free to copy and paste the following:

Junko Honda, Chieko Hirose, Masako Takahashi, Sonoka Hisaoka, Miyuki Kanematsu, Yoshimi Bando and Mitsunori Sasa (2011). 3-Dimensional CT Lymphography in Identifying the Sentinel Node in Breast Cancer, Radioisotopes - Applications in Bio-Medical Science, Prof. Nirmal Singh (Ed.), ISBN: 978-953-307-748-2, InTech, Available from: http://www.intechopen.com/books/radioisotopes-applications-in-bio-medical-science/3dimensional-ct-lymphography-in-identifying-the-sentinel-node-in-breast-cancer

\section{INTECH}

open science | open minds

\section{InTech Europe}

University Campus STeP Ri

Slavka Krautzeka 83/A

51000 Rijeka, Croatia

Phone: +385 (51) 770447

Fax: +385 (51) 686166

www.intechopen.com

\section{InTech China}

Unit 405, Office Block, Hotel Equatorial Shanghai

No.65, Yan An Road (West), Shanghai, 200040, China

中国上海市延安西路65号上海国际贵都大饭店办公楼 405 单元

Phone: +86-21-62489820

Fax: $+86-21-62489821$ 
(C) 2011 The Author(s). Licensee IntechOpen. This is an open access article distributed under the terms of the Creative Commons Attribution 3.0 License, which permits unrestricted use, distribution, and reproduction in any medium, provided the original work is properly cited. 